\title{
EVALUACIÓN DEL PROCESO DE REMOCIÓN DE Cr(VI) CON COMPOST DE RESIDUOS DE CULTIVO DE CLAVEL EN DISOLUCIONES ACUOSAS
}

\author{
Elianna Castillo*a, Claudia Vargas ${ }^{\text {a }}$, Pedro F. B. Brandão ${ }^{a}$, Jesús A. Ágreda ${ }^{a}$
}

\begin{abstract}
RESUMEN
En este trabajo se presenta un estudio para determinar el mecanismo del proceso de remoción de $\mathrm{Cr}$ (VI) de disoluciones acuosas, usando como adsorbente un compost obtenido de residuos del cultivo de clavel. En primer lugar, se determinó si la microbiota presente en el material afecta la remoción de Cr(VI). Para esto se realizaron medidas respirométricas y se evaluó la capacidad de remoción usando compost esterilizado y sin esterilizar. Adicionalmente, se analizó el compost antes y después de llevar a cabo el proceso de remoción de $\mathrm{Cr}(\mathrm{VI})$, mediante cuantificación de grupos funcionales ácidos y seguimiento cinético de $\mathrm{pH}$, conductividad y diferencia de potencial redox. Los resultados mostraron que, en las condiciones utilizadas, los microorganismos presentes en el compost no afectan el proceso de remoción de $\mathrm{Cr}(\mathrm{VI}) \mathrm{y}$ el mecanismo se puede describir mediante un modelo de adsorción acoplada a la reducción. Esta adsorción se debe principalmente a los grupos funcionales ácidos (carboxílicos y fenólico-hidroxílicos). Con esta información, se construyó un mecanismo de 7 pasos que describe satisfactoriamente el proceso de remoción estudiado. El conjunto de constantes cinéticas del mecanismo propuesto se optimizó usando el análisis de sensibilidad. Finalmente, el mecanismo propuesto se verificó mediante simulaciones numéricas, encontrando buena concordancia entre los datos experimentales y los simulados.
\end{abstract}

Palabras clave: Remoción de Cr(VI), compost, medidas respirométricas, medidas cinéticas, conductividad, diferencia de potencial redox, residuos de cultivo de clavel.

\section{EVALUATION OF Cr(VI) REMOVAL PROCESS IN AQUEOUS SOLUTIONS USING CARNATION CROP WASTE COMPOST}

\begin{abstract}
This paper presents a study that aimed to determine the mechanism of a process for $\mathrm{Cr}(\mathrm{VI})$ removal from aqueous solutions, using compost of carnation crop residues as the adsorbent. We assessed whether the microbiota present in the absorbent material affected the removal of $\mathrm{Cr}(\mathrm{VI})$ using respirometric measurements and evaluating the removal capacity using sterilized and not sterilized compost. Additionally, the compost was analysed before and after

a Grupo de estudios para la Remediación y Mitigación de Impactos Negativos al Ambiente - GERMINA, Departamento de Química, Facultad de Ciencias, Universidad Nacional de Colombia - Sede Bogotá, Av Cra 30 No. 45 - 03, Bogotá D.C., Código Postal 111321, Colombia.

*ecastillo@unal.edu.co


carrying out the $\mathrm{Cr}(\mathrm{VI})$ removal process, by quantification of the acidic functional groups and kinetic monitoring of $\mathrm{pH}$, conductivity and redox potential difference. The results showed that, under the conditions used, the microorganisms present in the compost did not affect the process of $\mathrm{Cr}(\mathrm{VI})$ removal and that the mechanism can be described by an adsorption model coupled to the reduction, were this adsorption is mainly done on the acidic functional groups (carboxylic and phenolic-hydroxylic). Base on this information, a mechanism of 7 reactions was constructed to describe the removal process. The kinetic constants for each reaction were optimized with a sensitivity analysis process. The proposed mechanism was verified by numerical simulations and a good agreement was found between experimental and simulated data.

Key words: Removal of $\mathrm{Cr}(\mathrm{VI})$, compost, respirometric measures, kinetic measurements, conductivity, redox potential difference, carnation crop waste.

\section{INTRODUCCIÓN}

Los sistemas de biorremediación son cada vez más utilizados para la remoción de metales pesados $^{1}$. Por ejemplo, la remoción de $\mathrm{Cr}(\mathrm{VI})$ de aguas contaminadas utilizando compost como adsorbente. El compost es un material con alto contenido de materia orgánica que usualmente se emplea para la recuperación y adecuación de suelos; además, se ha demostrado que también puede ser utilizado como adsorbente para la remoción de metales tóxicos ${ }^{2}$.

En trabajos anteriores del grupo de investigación ${ }^{3,4}$ se ha encontrado que el compost obtenido a partir de la estabilización de residuos del cultivo de claveles, producidos en la sabana de Bogotá, puede ser utilizado para remover $\mathrm{Cr}(\mathrm{VI})$ de disoluciones acuosas. En las condiciones óptimas encontradas ( $\mathrm{pH}$ 2,0; dosis de adsorbente $1 \mathrm{~g} / 100 \mathrm{~mL}$ de disolución; y tiempo de contacto $3 \mathrm{~h}$ ), la isoterma de adsorción se ajustó satisfactoriamente al modelo de Langmuir, encontrando una capacidad máxima de adsorción de $6,25 \mathrm{mg} / \mathrm{g}$, valor comparable con otros materiales adsorbentes de bajo costo. Además, se reportó una aproximación físico-química al mecanismo de dicha remoción, la cual está soportada en estudios de espectroscopia FTIR y microscopía SEM, antes y después del proceso de remoción ${ }^{3}$.

El mecanismo de remoción de Cr(VI) con estos materiales no es fácil de entender, en gran parte por la heterogeneidad del material. Por esta razón, existen pocos estudios al respecto los cuales se pueden resumir en las siguientes etapas: 1) mecanismo de reducción directa: en este caso se propone que el $\mathrm{Cr}(\mathrm{VI})$ se reduce a $\mathrm{Cr}(\mathrm{III})$, el cual luego es expulsado hacia la fase acuosa; 2) mecanismo de "adsorción acoplado a la reducción", en este caso se proponen 3 etapas: (i) el $\mathrm{Cr}(\mathrm{VI})$ se enlaza a la superficie del bioadsorbente mediante atracción electrostática con los grupos cargados positivamente; (ii) el $\mathrm{Cr}$ (VI) se reduce a $\mathrm{Cr}$ (III) debido a los grupos electro-donores adyacentes al Cr(VI) adsorbido; y (iii) el Cr(III) generado sobre la superficie del bioadsorbente es expulsado a la fase acuosa debido a la repulsión electrónica entre los grupos cargados positivamente de la superficie y el $\mathrm{Cr}$ (III) generado ${ }^{5}$. Por otro lado, diferentes autores mostraron, mediante análisis espectroscópicos, que el $\mathrm{Cr}$ (III) que se genera después de la reducción del $\mathrm{Cr}(\mathrm{VI})$ puede quedar retenido en la superficie del material ${ }^{6}$. 
El objetivo de este trabajo es profundizar en los detalles del mecanismo de remoción del Cr usando compost como material adsorbente, en condiciones previamente optimizadas y reportadas $^{3}$. En busca de este objetivo, se realizaron estudios, biológicos y fisicoquímicos, para establecer un mecanismo más detallado. Se evaluó, entonces, si la microbiota presente en el material afecta la remoción de $\mathrm{Cr}(\mathrm{VI})$; para esto, se realizaron medidas respirométricas y se evaluó la capacidad de remoción usando compost esterilizado y sin esterilizar. Posteriormente, se llevó a cabo un estudio cinético del proceso de remoción mediante el seguimiento simultáneo de $\mathrm{pH}$, conductividad y diferencia de potencial redox. El mecanismo propuesto se verificó mediante simulaciones numéricas, las cuales presentaron buena concordancia con los datos experimentales.

\section{PARTE EXPERIMENTAL}

\section{Caracterización del compost}

Elmaterial adsorbente usado para todos los ensayos fue un compost comercial(Ferticompost ${ }^{\circledR}$ ), comercializado por la empresa Compostar Ltda., y elaborado a partir de la estabilización de los residuos de clavel generados por la empresa Flores de Serrezuela S.A., Bogotá, Colombia. Este material, se secó a temperatura ambiente y se realizó su caracterización. Se determinaron los parámetros fisicoquímicos exigidos en la Norma Técnica Colombiana NTC5167 del 2004 (pH, conductividad, densidad, porcentaje de humedad, contenido de cenizas, nitrógeno, carbono orgánico oxidable y capacidad de intercambio catiónico). La composición elemental se determinó mediante fluorescencia de rayos-X (FRX), gracias a la ayuda del Servicio Geológico Colombiano* y utilizando un instrumento AXIOS MINERALS (Panalytical). La evaluación de las características texturales del compost se realizó a partir de isotermas de adsorción-desorción de nitrógeno a $77^{\circ} \mathrm{K}$. El área superficial del compost se estimó mediante el modelo BET ${ }^{7}$.

\section{Efecto de la esterilización del compost en la remoción de $\mathrm{Cr}(\mathrm{VI})$}

Para evaluar si los microorganismos vivos del biomaterial presentan efectos metabólicos en el proceso de remoción de $\mathrm{Cr}(\mathrm{VI})$, se construyó una isoterma de adsorción con compost esterilizado en autoclave durante 20 minutos a $121^{\circ} \mathrm{C}$. Además, y mediante estudios respirométricos, se comprobó la actividad microbiológica del compost antes y después del proceso de remoción. La isoterma de adsorción se realizó en el intervalo de concentraciones iniciales de $\mathrm{Cr}(\mathrm{VI})$ de $15 \mathrm{mg} / \mathrm{L}$ a $200 \mathrm{mg} / \mathrm{L}$, en las mejores condiciones experimentales encontradas en un estudio previo3: relación de $100 \mathrm{~mL}$ de disolución por cada gramo de compost (con tamaño de partícula menor a $180 \mu \mathrm{m}$ ), tiempo de agitación de $3 \mathrm{~h}$ a $36 \mathrm{rpm}$ y $\mathrm{pH}$ inicial de 2,0 . Los resultados se presentan en términos de $\%$ de remoción usando la relación:

$$
\% \text { de Remoción }=\frac{\left(C_{0}-C_{\mathrm{e}}\right)}{C_{0}} * 100
$$

http://www2.sgc.gov.co/ 
En donde, $C_{0}$ y $C_{\mathrm{e}}$ son, respectivamente, las concentraciones iniciales y de equilibrio de $\mathrm{Cr}(\mathrm{VI})$.

\section{Medidas respirométricas}

Las medidas respirométricas se realizaron siguiendo la metodología de Barrena y colaboradores $^{8}$, la cual se describe brevemente a continuación: $10 \mathrm{~g}$ de compost, previamente humectada hasta alcanzar una humedad cercana al $60 \%$ e incubada durante 24 horas a $25^{\circ} \mathrm{C}$, fue colocada en un erlenmeyer de $500 \mathrm{~mL}$, el cual contenía un recipiente con $10 \mathrm{~mL}$ de $\mathrm{NaOH} 0,04 \mathrm{M}$, durante $24 \mathrm{~h}$ a $25^{\circ} \mathrm{C}$; el $\mathrm{CO}_{2}$ producido se determinó mediante titulación de la muestra de $\mathrm{NaOH}$ con $\mathrm{HCl} \mathrm{0,04} \mathrm{M}$.

\section{Cuantificación de grupos funcionales ácidos}

La cuantificación de los grupos funcionales ácidos presentes en el compost, antes y después de remover $\mathrm{Cr}(\mathrm{VI})$, se realizó mediante la determinación de los diferentes tipos de acidez presentes en el compost: acidez total, acidez proveniente de grupos carboxilícos y acidez proveniente de los grupos fenólicos e hidroxílicos ${ }^{9}$.

La acidez total se determinó agitando durante $24 \mathrm{~h}, 150 \mathrm{mg}$ de muestra con $20 \mathrm{~mL}$ de $\mathrm{Ba}(\mathrm{OH})_{2}$ 0,2 M. Una vez finalizado este período, las muestras se filtraron usando papel Watman $45 \mathrm{y}$ el filtrado se tituló potenciométricamente con $\mathrm{HCl}$ 0,40 $\mathrm{M}$ hasta alcanzar $\mathrm{pH} 8,4$. La acidez total se calculó mediante la siguiente expresión:

$$
\text { Acidez Total }\left(\frac{m m o l}{g}\right)=\frac{\left(V_{B}-V_{m}\right) * M_{H C l}}{P_{w}}
$$

donde, $V_{B}$ es el volumen gastado en la titulación del blanco $(\mathrm{mL}), V_{m}$ es el volumen gastado en la titulación de la muestra $(\mathrm{mL}), M_{H C l}$ es la concentración molar de $\mathrm{HCl}$ y $P_{w}$ es el peso de la muestra $(\mathrm{g})$.

La determinación de la acidez proveniente de los grupos carboxílicos se realizó agitando 150 $\mathrm{mg}$ de muestra con $10 \mathrm{~mL}$ de $\mathrm{Ca}\left(\mathrm{CH}_{3} \mathrm{COO}\right)_{2} 1 \mathrm{M}$ en $40 \mathrm{~mL}$ de agua desionizada durante $24 \mathrm{~h}$. Las muestras se filtraron usando papel Watman 42 y el filtrado se tituló con $\mathrm{NaOH} 0,1 \mathrm{M}$. La acidez proveniente de los grupos carboxílicos se calculó usando la ecuación:

$$
\text { Acidez grupos carboxílicos }\left(\frac{m m o l}{g}\right)=\frac{\left(V_{m}-V_{B}\right) * M_{N a O H}}{P_{w}}
$$

en donde, $V_{B}$ es el volumen gastado en la titulación del blanco $(\mathrm{mL}), V_{m}$ es el volumen gastado en la titulación de la muestra $(\mathrm{mL}), M_{\mathrm{NaOH}}$ es la concentración molar del $\mathrm{NaOH}$ y $P_{w}$ es el peso de la muestra (g). La acidez proveniente de los grupos fenólicos e hidroxílicos se calculó como la diferencia de la acidez total y de la acidez proveniente de los grupos carboxílicos.

\section{Estudio cinético: $\mathbf{p H}$, conductividad y diferencia de potencial redox}

Los estudios cinéticos se hicieron tomando como referencia las condiciones optimizadas en un trabajo anterior ${ }^{3}$. Para el registro de los datos se utilizó un potenciostato eDAQ e-corder 
210 conectado a una interface pH \& ISE IsoPod EP 353, sistema con el cual se midió el $\mathrm{pH}$ y la diferencia de potencial redox. La conductividad se siguió usando una interface conductivity IsoPod EP 357.

\section{Reactivos y disoluciones}

La calibración del electrodo de $\mathrm{pH}$ se realizó utilizando reguladores de $\mathrm{pH}$ 7,00 y 4,00 \pm 0,01 (Merck). La calibración del electrodo redox se llevó a cabo utilizando las mismas disoluciones de $\mathrm{pH} 7,00$ y $4,00 \pm 0,01$, pero ahora saturadas con quinhidrona. El electrodo de conductividad se calibró con disoluciones de $\mathrm{KCl} 0,0100 \pm 0,0001 \mathrm{M}$ y $0,0010 \pm 0,0001 \mathrm{M}$. Ensayos para el estudio cinético

El estudio cinético del proceso de remoción se realizó siguiendo el esquema que se presentan en la tabla 1.

Tabla 1. Experimentos usados para el estudio cinético de adsorción de $\mathrm{Cr}(\mathrm{VI})$ sobre compost.

\begin{tabular}{ccccc}
\hline Experimento & $\begin{array}{c}\text { Agua desionizada } \\
(50 \mathrm{~mL})\end{array}$ & $\begin{array}{c}\text { Disolución de Cr(VI) } \\
50 \mathrm{mg} / \mathrm{L}(50 \mathrm{~mL})\end{array}$ & $\begin{array}{c}\text { Compost } \\
(0,5 \mathrm{~g})\end{array}$ & $\begin{array}{c}\mathrm{pH} \text { inicial } \\
\text { ajustado a 2,0 }\end{array}$ \\
\hline 1 & $\sqrt{ }$ & & $\sqrt{ }$ & $\sqrt{ }$ \\
2 & $\sqrt{ }$ & $\sqrt{ }$ & $\sqrt{ }$ & $\sqrt{ }$ \\
3 & & $\sqrt{ }$ & $\sqrt{ }$ & \\
4 & & $\sqrt{ }$ & $\sqrt{ }$ & $\sqrt{ }$ \\
5 & &
\end{tabular}

\section{Ajustes no lineales y simulaciones}

Las regresiones no lineales se realizaron usando el software $\mathrm{R}$ ( $\mathrm{R}$ versión 2.13.0 Copyright ${ }^{\circ}$ 2011, The R Foundation for Statistical Computing). Las simulaciones numéricas se realizaron usando un programa de computadora escrito en FORTRAN, que usa la subrutina de integración LSODE ${ }^{10}$.

\section{RESULTADOS Y DISCUSIÓN}

\section{Caracterización del compost}

Los resultados de la caracterización fisicoquímica del compost Ferticompost $₫$ se presentan en la tabla 2. Los resultados de esta caracterización se compararon con la norma técnica colombiana NTC5167, en la que se estipulan las condiciones que debe cumplir un material usado para la enmienda de suelos en Colombia. Al evaluar los resultados se puede inferir que el compost Ferticompost ${ }^{\circledR}$ cumple con los parámetros establecidos para ser usado como enmienda orgánica en Colombia. 
Tabla 2. Caracterización fisicoquímica del compost.

\begin{tabular}{lccc}
\hline \multicolumn{1}{c}{ Parámetro } & Unidades & Resultado & NTC 5167 \\
\hline Humedad & $\%$ & $23,1 \pm 0,7$ & Máximo 35 \\
Cenizas & $\%$ & $44,8 \pm 1,8$ & Máximo 60 \\
Carbono orgánico oxidable & $\%$ & $19,9 \pm 1,7$ & Mínimo 15 \\
Capacidad de intercambio catiónico & $\mathrm{meq} / 100 \mathrm{~g}$ & $49,4 \pm 0,7$ & Mínimo 30 \\
Densidad & $\mathrm{g} / \mathrm{cm}^{3}$ & $0,33 \pm 0,01$ & Máximo 0,6 \\
$\mathrm{pH}$ & - & $6,86 \pm 0,2$ & $4-9$ \\
$\mathrm{P}_{2} \mathrm{O}_{5}$ & $\%$ & $1,04 \pm 0,08$ & - \\
$\mathrm{Nitrógeno}$ & $\%$ & $0,89 \pm 0,07$ & - \\
$\mathrm{C} / \mathrm{N}$ & - & 21,89 & - \\
Capacidad de retención de agua & $\%$ & $137,5 \pm 0,4$ & Mínimo 100 \\
\hline
\end{tabular}

A partir de los datos de la isoterma de adsorción-desorción de $\mathrm{N} 2$ a $77^{\circ} \mathrm{K}$, se obtuvo un área superficial de $6,7 \mathrm{~m}^{2} / \mathrm{g}$ del compost. Comparando este valor con el reportado para otros adsorbentes usados en la remoción de $\mathrm{Cr}(\mathrm{VI})$, se concluye que, a pesar de ser un valor bajo, presenta un área superficial mayor que materiales adsorbentes similares como por ejemplo la fibra de coco $\left(0,8 \mathrm{~m}^{2} / \mathrm{g}\right)$, residuos de uva $\left(5,3 \mathrm{~m}^{2} / \mathrm{g}\right)$ o las cenizas $\left(6,4 \mathrm{~m}^{2} / \mathrm{g}\right)^{3}$.

\section{Actividad metabólica de los microorganismos en el compost}

Para evaluar el efecto de la actividad metabólica de los microorganismos presentes en el compost en la remoción de $\mathrm{Cr}(\mathrm{VI})$, se llevó a cabo el proceso de remoción a diferentes concentraciones iniciales del metal usando el compost esterilizado y sin esterilizar. En la figura 1, se puede observar que la remoción usando compost esterilizado es similar al compost sin esterilizar, lo cual sugiere que no hay un efecto metabólico de los microorganismos presentes en el compost que contribuya al proceso de remoción del metal. Por lo tanto, se considera que el proceso de remoción es netamente fisicoquímico. Este resultado difiere de otros autores ${ }^{11}$, quienes mostraron que la remoción de $\mathrm{Cr}(\mathrm{VI})$ está ligada con la actividad microbiológica presente en el compost. Estos resultados, posiblemente, son diferentes debido a que nuestros ensayos de remoción se realizaron a $\mathrm{pH}$ inicial de 2,0 mientras que los realizados por esos autores fueron con el compost a $\mathrm{pH} 8,4$. Esto implica que a $\mathrm{pH} 2,0$ los microorganismos ya estarían inactivos, inclusive en el compost sin esterilizar.

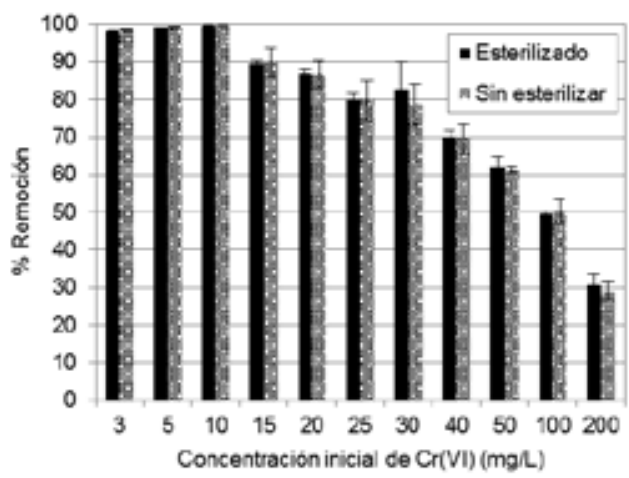

Figura 1. Evaluación del efecto del uso de compost esterilizado o sin esterilizar para la remoción de distintas concentraciones de $\mathrm{Cr}(\mathrm{VI})$. Condiciones iniciales: $\mathrm{pH}$ 2,0; $1 \mathrm{~g}$ de compost por cada $100 \mathrm{~mL}$ de disolución; agitación circular a 36 rpm y tiempo de agitación de 3 horas. 


\section{Pruebas respirométricas}

Las medidas respirométricas están relacionadas con la actividad microbiológica de los microorganismos presentes en el compost. En los ensayos realizados se encontraron valores de índices repirométricos de 0,48 $\pm 0,07$ y 0,21 $\pm 0,07\left(\mathrm{mg} \mathrm{CO}_{2} / \mathrm{g}\right)$, antes y después del proceso de remoción respectivamente, lo cual sugiere que los microorganismos presentes en el compost fueron afectados negativamente por la presencia del metal, indicando una disminución en la actividad metabólica de los microorganismos después del proceso de remoción de $\mathrm{Cr}(\mathrm{VI})$.

\section{Cuantificación de grupos funcionales ácidos}

Los resultados obtenidos para la cuantificación de grupos ácidos (grupos carboxílicos, fenólicos e hidroxílicos) se presentan en la tabla 3.

Tabla 3. Cuantificación de grupos ácidos del compost antes y después de la remoción de $\mathrm{Cr}(\mathrm{VI})$.

\begin{tabular}{cccc}
\hline \multirow{2}{*}{$\begin{array}{c}\text { Remoción de } \\
\text { Cr(VI) }\end{array}$} & $\begin{array}{c}\text { Acidez } \\
\text { total }\end{array}$ & $\begin{array}{c}\text { Grupos de acidez }(\mathrm{mmol} / \mathrm{g}) \\
\text { carboxílicos }\end{array}$ & $\begin{array}{c}\text { Grupos } \\
\text { fenólicos-hidroxílicos }\end{array}$ \\
\cline { 2 - 4 } ANTES & $2,60 \pm 0,13$ & $0,08 \pm 0,03$ & $2,52 \pm 0,14$ \\
DESPUÉS & $1,45 \pm 0,56$ & $0,47 \pm 0,03$ & $0,97 \pm 0,55$ \\
\hline
\end{tabular}

Se observa que el compost estudiado tiene mayor cantidad de grupos fenólicos que carboxílicos, tendencia que se mantiene antes y después de la remoción del Cr(VI) y a pesar del aumento significativo de los grupos carboxílicos luego de la remoción del metal. De otro lado, el aumento de los grupos carboxílicos, tras la remoción del $\mathrm{Cr}(\mathrm{VI})$, es consistente con la reducción del metal a $\mathrm{Cr}(\mathrm{III})$, hecho que ya fue mencionado anteriormente y que será incluido en el mecanismo que se propone más adelante. Estos resultados son comparables con trabajos previos ${ }^{6,7}$ en donde se ha demostrado, con estudios de FT-IR, que el compost antes y después de la remoción del Cr(VI), presenta interacciones con el metal a través de sus grupos funcionales, principalmente grupos fenólicos e hidroxílicos.

Además, se observa una disminución en la acidez total del compost cuando este está cargado con $\mathrm{Cr}(\mathrm{VI})$, lo cual evidencia que este metal se adsorbe sobre este tipo de grupos funcionales como lo han establecido otros autores mediante medidas espectroscópicas6. A partir de estos resultados, se decidió estudiar el efecto del $\mathrm{pH}$, la diferencia de potencial redox y la conductividad durante el proceso de remoción del $\mathrm{Cr}(\mathrm{VI})$ con el compost en cuestión. Todo esto, con el objetivo de encontrar pistas que ayuden a elucidar el mecanismo de remoción del Cr(VI) con el compost.

\section{Estudio cinético: mediciones de pH}

El comportamiento del $\mathrm{pH}$, para los 5 experimentos descritos en la tabla 1 de la sección experimental, se presenta en la figura 2. Se pueden observar tres tendencias: 1) dos disoluciones cuyo $\mathrm{pH}$ inicial no fue ajustado a 2,0 (compost en agua y compost en agua con $\mathrm{Cr}(\mathrm{VI})$ ): estas tendencias muestran la naturaleza ligeramente básica del compost y, además, 
se puede observar que cuando al compost en agua (trazo superior de la figura) se le agrega $\mathrm{Cr}(\mathrm{VI})$, el $\mathrm{pH}$ desciende un poco debido a la naturaleza ácida de las disoluciones acuosas de cromo; 2) disolución de $\mathrm{Cr}(\mathrm{VI})$ cuyo $\mathrm{pH}$ inicial se ajustó a 2,0: en este caso se observa que el $\mathrm{pH}$ disminuye, tendiendo a tiempos largos a un valor alrededor de $\mathrm{pH} \mathrm{1,9;} \mathrm{y} \mathrm{3)} \mathrm{disoluciones}$ que tienen compost con y sin cromo y a las que se les ajustó el $\mathrm{pH}$ inicial a un valor de 2,0: en este caso el $\mathrm{pH}$ tiende a aumentar con el tiempo.

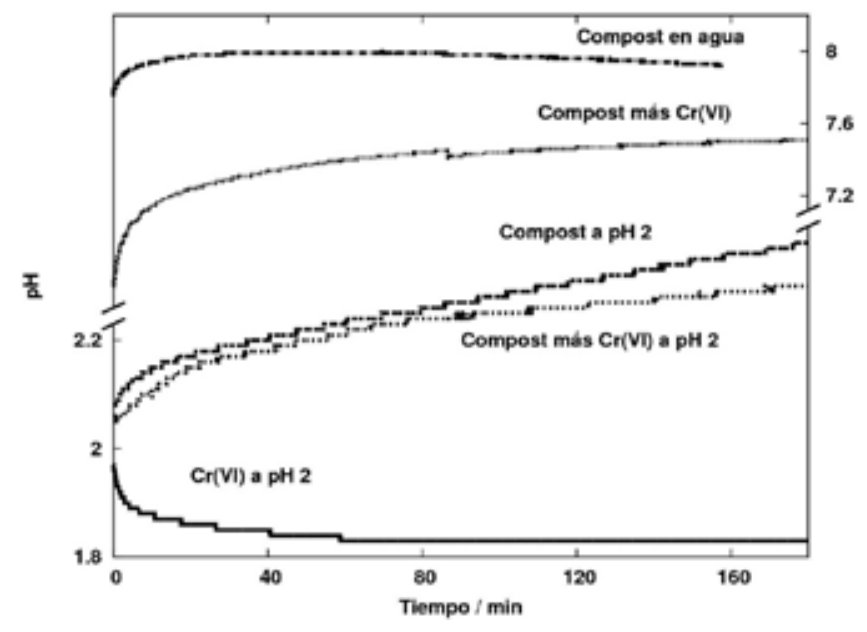

Figura 2. Cinética de la adsorción de $\mathrm{Cr}(\mathrm{VI})$ sobre compost. Medidas de $\mathrm{pH}$ en función del tiempo para los 5 experimentos descritos en la tabla 1. Para obtener mayor claridad en la figura, los datos de la disolución de compost a $\mathrm{pH} 2,0, \sin \mathrm{Cr}(\mathrm{VI})$, fueron desplazados en $-0,04$ unidades de $\mathrm{pH}$.

Estos dos últimos conjuntos de datos experimentales se ajustaron a una cinética de orden 2, utilizando una regresión no lineal. Los resultados del ajuste se muestran en la tabla 4.

Tabla 4. Ajuste no lineal a una cinética de orden 2 de los datos experimentales de $\left[\mathrm{H}_{3} \mathrm{O}^{+}\right]$en función del tiempo.

\begin{tabular}{|c|c|c|c|c|}
\hline \multicolumn{3}{|c|}{ Ecuación a la que se ajustaron los datos: } & \multicolumn{2}{|c|}{$\left.\mathrm{O}^{+}\right]=\frac{\left[\mathrm{H}_{3} \mathrm{O}^{+}\right]_{0}\left(\left[\mathrm{H}_{3} \mathrm{O}^{+}\right]_{0}-\mathbf{A}_{0}\right)}{(-1)}$} \\
\hline & \multicolumn{2}{|c|}{ Compost $\mathrm{pH}$ inicial 2,0 } & \multicolumn{2}{|c|}{ Compost más $\mathrm{Cr}(\mathrm{VI}) \mathrm{pH}$ inicial 2,0 } \\
\hline & Valor & Error estándar & Valor & Error estándar \\
\hline $\mathrm{A}_{0} / \mathrm{mM}$ & 5,47 & 0,03 & 3,614 & 0,004 \\
\hline$\left[\mathrm{H}_{3} \mathrm{O}^{+}\right]_{0} / \mathrm{mM}$ & 7,947 & 0,004 & 8,603 & 0,004 \\
\hline $\mathrm{k} / \mathrm{mM}^{-1} \mathrm{~s}^{-1}$ & $9,75 \times 10^{-4}$ & $8 \times 10^{-6}$ & $2,861 \times 10^{-3}$ & $9 \times 10^{-6}$ \\
\hline \multicolumn{2}{|c|}{$\begin{array}{c}\text { Error estándar residual } \\
\text { Tolerancia en la convergencia }\end{array}$} & $\begin{array}{c}0,1 \\
6 \times 10^{-6}\end{array}$ & \multicolumn{2}{|c|}{$\begin{array}{c}0,09 \\
5 \times 10^{-6}\end{array}$} \\
\hline
\end{tabular}

Estos dos experimentos, a $\mathrm{pH}$ inicial de 2,0 con y sin $\mathrm{Cr}(\mathrm{VI})$, resultan importantes ya que se ha demostrado, y se confirmará más adelante, que la protonación del compost es un 
requisito que debe ser cumplido antes de que el $\mathrm{Cr}(\mathrm{VI})$ se pueda adsorber ${ }^{12}$. Así, el proceso de adsorción de $\mathrm{Cr}(\mathrm{VI})$ sobre el compost, obtenido de residuos del cultivo de claveles, puede ser representado por una reacción de segundo orden entre el $\mathrm{Cr}(\mathrm{VI})$ y la superficie del compost, el cual ocurre siempre y cuando el compost esté previamente protonado. Asimismo, como se muestra en la tabla 4, la protonación del compost es un proceso de segundo orden. Estos hechos experimentales se pueden representar con las siguientes ecuaciones:

$$
\begin{gathered}
\text { Compost }+\mathrm{H}_{3} \mathrm{O}^{+} \rightleftharpoons \text { Compost } \cdots \mathrm{H}+\mathrm{H}_{2} \mathrm{O} \\
\text { Compost } \cdots \mathrm{H}+\mathrm{Cr}(\mathrm{VI}) \rightleftharpoons \text { Compost } \cdots \mathrm{H} \cdots \mathrm{Cr}(\mathrm{VI})
\end{gathered}
$$

\section{Estudio cinético: mediciones de conductividad y potencial redox}

La conductividad en función del tiempo de la disolución de $\mathrm{Cr}(\mathrm{VI})$ en contacto con el compost a $\mathrm{pH}$ 2,0 sigue la misma tendencia observada para la concentración del ión hidronio, esto es, disminuye en función del tiempo (figura 3a). Este comportamiento se puede explicar en virtud de que el ion $\mathrm{H}_{3} \mathrm{O}^{+}$es la especie en disolución acuosa con mayor conductividad molar específica, por lo que el cambio en su concentración tiene efecto definitivo sobre la conductividad de la disolución.
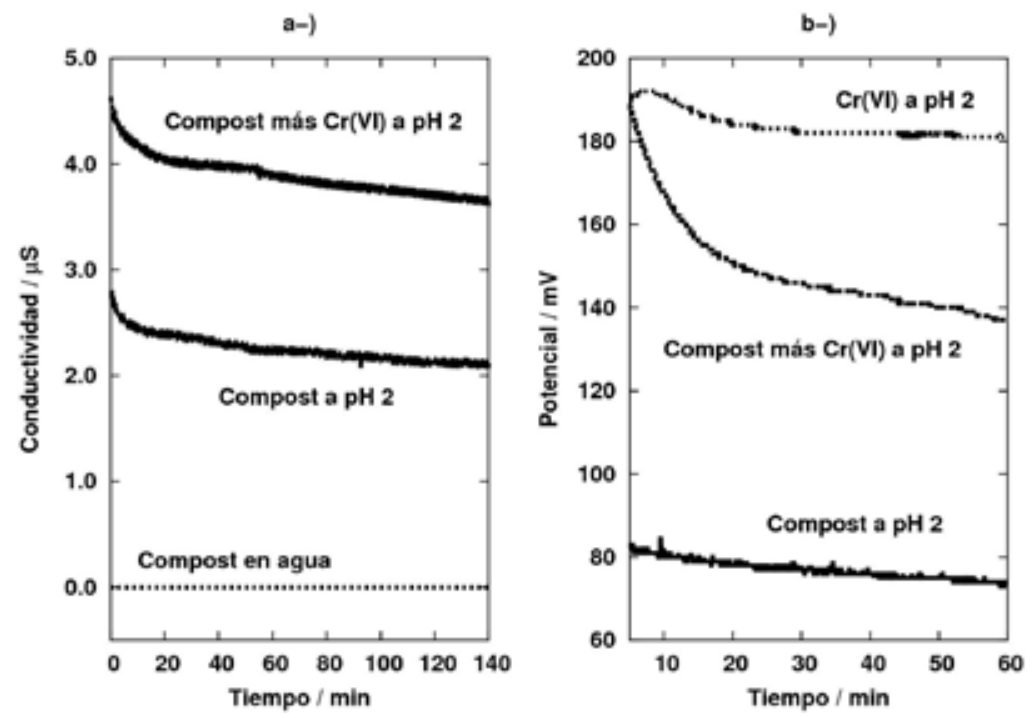

Figura 3. Cinética de la adsorción de Cr(VI) sobre compost. Medidas de: a) conductividad en función del tiempo y b) diferencia de potencial redox en función del tiempo.

Por otro lado, pueden existir especies conductoras en disolución, liberadas a la misma por el compost. En la tabla 5 se presentan los resultados obtenidos de la composición elemental del compost evaluado, empleando FRX. Los metales y sus aniones asociados pueden, eventualmente, pasar a la disolución y contribuir a la conductividad de la misma. 
Tabla 5. Composición elemental del compost utilizado para el estudio de remoción de $\mathrm{Cr}(\mathrm{VI})$ de aguas contaminadas. El análisis se realizó utilizando FRX.

\begin{tabular}{cc|cc|cc}
\hline $\begin{array}{c}\text { Elemento } \\
\text { expresado } \\
\text { como óxido }\end{array}$ & $\begin{array}{c}\text { Contenido } \\
\text { porcentual } \\
(\%)\end{array}$ & $\begin{array}{c}\text { Elemento } \\
\text { expresado } \\
\text { como óxido }\end{array}$ & $\begin{array}{c}\text { Contenido } \\
\text { porcentual } \\
(\%)\end{array}$ & $\begin{array}{c}\text { Elemento } \\
\text { expresado } \\
\text { como óxido }\end{array}$ & $\begin{array}{c}\text { Contenido } \\
\text { porcentual } \\
(\%)\end{array}$ \\
\hline $\mathrm{Al}_{2} \mathrm{O}_{3}$ & 6,23 & $\mathrm{MgO}$ & 1,64 & $\mathrm{SrO}$ & 0,042 \\
$\mathrm{BaO}$ & 0,025 & $\mathrm{Mn}_{3} \mathrm{O}_{4}$ & 0,133 & $\mathrm{TiO}_{2}$ & 0,23 \\
$\mathrm{CaO}$ & 6,85 & $\mathrm{Na}_{2} \mathrm{O}$ & 0,79 & $\mathrm{~V}_{2} \mathrm{O}_{5}$ & 0,008 \\
$\mathrm{Cr}_{2} \mathrm{O}_{3}$ & 0,002 & $\mathrm{NiO}$ & 0,001 & $\mathrm{ZnO}$ & 0,024 \\
$\mathrm{CuO}$ & 0,034 & $\mathrm{P}_{2} \mathrm{O}_{5}$ & 2,153 & $\mathrm{ZrO}_{2}$ & 0,01 \\
$\mathrm{Fe}_{2} \mathrm{O}_{3}$ & 1,37 & $\mathrm{SiO}_{2}$ & 40,78 & - & - \\
$\mathrm{K}_{2} \mathrm{O}$ & 1,64 & $\mathrm{SO}_{3}$ & 1,17 & - & - \\
\hline
\end{tabular}

La presencia de diferentes iones en disolución, hace difícil asociar las medidas de conductividad con una especie en particular, dado que las medidas de conductividad no son selectivas. Como consecuencia de esto, los registros de conductividad no mostraron un ajuste aceptable a cinéticas de orden 2 . Sin embargo, como lo muestra la figura $3 a$, la tendencia de la conductividad a disminuir con el tiempo es consistente con las expresiones descritas previamente sobre la protonación del compost, dado que en ellas se muestra que las especies cargadas eléctricamente (particularmente el ión $\mathrm{H}_{3} \mathrm{O}^{+}$) son eliminadas de la disolución, lo que lleva a que la conductividad disminuya.

En cuanto a las lecturas de la diferencia de potencial redox, los resultados mostraron que el Cr(VI) se reduce por acción del compost, como se puede observar en la figura 3b. Estos resultados indican que la reducción del $\mathrm{Cr}(\mathrm{VI})$ se lleva a cabo en la superficie protonada del compost, como se ha mostrado en otros estudios de reducción de $\mathrm{Cr}(\mathrm{VI})$ en otros biomateriales en condiciones ácidas ${ }^{13}$. Sin embargo, al igual que en el caso de las medidas de conductividad, los registros de diferencia de potencial redox no se pueden asociar a una sola especie en particular, por esta razón, no hubo un buen ajuste a cinéticas de orden 2 .

\section{Propuesta de mecanismo de remoción}

Basados en la evidencia experimental anterior, a saber: la cinética de segundo orden para el cambio de $\left[\mathrm{H}_{3} \mathrm{O}^{+}\right]$en función del tiempo, reducción del $\mathrm{Cr}(\mathrm{VI})$ sobre el compost y cinética de segundo orden para la adsorción del cromo sobre el compost; y considerando la información disponible en la literatura, a continuación se propone el mecanismo que se presenta en la tabla 6 para la adsorción de $\mathrm{Cr}(\mathrm{VI})$ sobre el compost de residuos de claveles partiendo de un $\mathrm{pH}$ de 2,0 . 
Tabla 6. Mecanismo de reacción propuesto para la adsorción de $\mathrm{Cr}(\mathrm{VI})$ sobre compost elaborado con residuos del cultivo de clavel de la sabana de Bogotá. El pH inicial se ajustó a 2,0.

\begin{tabular}{clc}
\hline Reacción & Constante cinética & Número \\
\hline$S_{\text {red }}+H_{3} O^{+} \rightleftharpoons S_{\text {red }} \cdots H+H_{2} O$ & $\mathrm{k}_{1}=8,0 \times 10^{-3} ; \mathrm{k}_{-1}=1,9 \times 10^{-3}$ \\
$S_{\text {red }} \cdots H+C r(V I) \rightleftharpoons S_{\text {red }} \cdots H \cdots C r(V I)$ & $\mathrm{k}_{2}=2,810^{3} ; \mathrm{k}_{-2}=1,0 \times 10^{-3}$ \\
$S_{\text {red }} \cdots H \cdots C r(V I) \rightleftharpoons S_{\text {ox }} \cdots H \cdots C r(I I I)$ & $\mathrm{k}_{3}=1,9 \times 10^{3} ; \quad \mathrm{k}_{-3}=1,0 \times 10^{-3}$ \\
$S_{\text {ox }} \cdots H \cdots \operatorname{Cr}(I I I) \rightleftharpoons S_{\text {ox }} \cdots H+\operatorname{Cr}(I I I)$ & $\mathrm{k}_{4}=1,0 \times 10^{-3} ; \mathrm{k}_{-4}=1,0 \times 10^{-3}$ \\
$S_{\text {red }} \cdots H+C r(V I) \rightleftharpoons S_{\text {ox }} \cdots H+C r(I I I)$ & $\mathrm{k}_{5}=1,0 \times 10^{2} ; \quad \mathrm{k}_{-5}=1,0$
\end{tabular}

Nota: la letra $S$ representa la superficie del compost en diversas formas: reducida, $S_{\text {red }}$; reducida y protonada, $S_{\text {red }} \cdots H$; con $\mathrm{Cr}(\mathrm{VI})$ adsorbido, $S_{\text {red }} \cdots H \cdots \mathrm{Cr}(\mathrm{VI})$; oxidada y con $\mathrm{Cr}(\mathrm{III})$ adsorbido, $S_{o x} \cdots H \cdots C r(I I I)$; oxidada, $S_{o x} \cdots H$; una superficie específica para la adsorción del $\operatorname{Cr}(\mathrm{III}), S_{2}$; superficie específica con Cr(III) adsorbido, $S_{2} \cdots C r(I I I)$; y la superficie del compost degradada, $S_{D}$.

El mecanismo de la tabla 6 condensa los hechos experimentales encontrados en este trabajo con la información obtenida de la literatura. A continuación, se describe cada paso del mecanismo propuesto, sustentando cada uno de ellos, ya sea con los resultados experimentales obtenidos o con referencias pertinentes:

1. La superficie del compost debe protonarse antes de que pueda ocurrir la adsorción del $\mathrm{Cr}(\mathrm{VI})$ : sustentado por los resultados experimentales del presente trabajo, así como por lo reportado en literatura ${ }^{12}$; reacción número (1).

2. La protonación del compost sigue una cinética de segundo orden: corroborado experimentalmente en este trabajo (tabla 4) y reportado en la literatura ${ }^{3}$; reacción número (1).

3. La adsorción del Cr(VI) sobre el compost sigue una cinética de orden 2: sustentado por la parte experimental de este trabajo (tabla 4) y reacción 2 reportada anteriormente 3 .

4. El Cr(VI) se reduce por acción del compost: sustentado por la parte experimental de este trabajo (Figura $3 b$ ) y por lo reportado en la literatura ${ }^{14}$; reacciones número (3) y (5).

5. El Cr(VI) se puede reducir, sin necesidad de adsorberse: reportado en la literatura ${ }^{13}$; reacción número (5).

6. El Cr(III) generado por la reducción del $\mathrm{Cr}(\mathrm{VI})$ pasa a la disolución, permitiendo las reacciones números (4) y (5), y se puede re-adsorber sobre el compost en un tipo de sitios activos diferentes a los iniciales: reportado en la referencia ${ }^{15}$; reacción número (6).

7. La reacción 7 se incluye para tener en cuenta el marcado decremento de la concentración de $\mathrm{H}_{3} \mathrm{O}^{+}$que se observó experimentalmente, y que no es posible describir con las primeras 6 reacciones. Este último paso, se puede entender como la degradación de la superficie del compost debida a la acidez del medio.

\section{Simulaciones numéricas del mecanismo propuesto}

El anterior mecanismo fue puesto a prueba para saber hasta qué punto podía describir el comportamiento experimental observado. El proceso se realizó aplicando valores iniciales 
estimados a las constantes cinéticas de las 7 reacciones del mecanismo los cuales, además, se optimizaron usando un proceso de análisis de sensibilidad ${ }^{16}$. Este análisis permitió identificar que las constantes cinéticas $\mathrm{k}_{1} \mathrm{y} \mathrm{k}_{-1}$ del primer proceso (la protonación del compost, reacción número (1)), la constante $\mathrm{k}_{2}$ de la reacción número (2) en el sentido directo (la adsorción del cromo hexavalente), y la constante $\mathrm{k}_{7}$ del proceso irreversible de degradación del compost por acción del medio ácido (reacción número (7)), son las que mayor efecto tienen sobre el comportamiento de las simulaciones.

Finalmente, el análisis de sensibilidad acoplado a un proceso de optimización simplex, nos permitió ajustar los valores de las constantes cinéticas de todas las reacciones del mecanismo propuesto, obteniendo los valores que se muestran en la tabla 7. Esto generó una buena concordancia entre los resultados experimentales y las simulaciones, como se puede observar en la figura 4 , en la que se presenta la concentración de los iones $\mathrm{H}_{3} \mathrm{O}^{+}$ medidos experimentalmente, y aquellos obtenidos mediante la simulación numérica usando el mecanismo propuesto de la tabla 6.

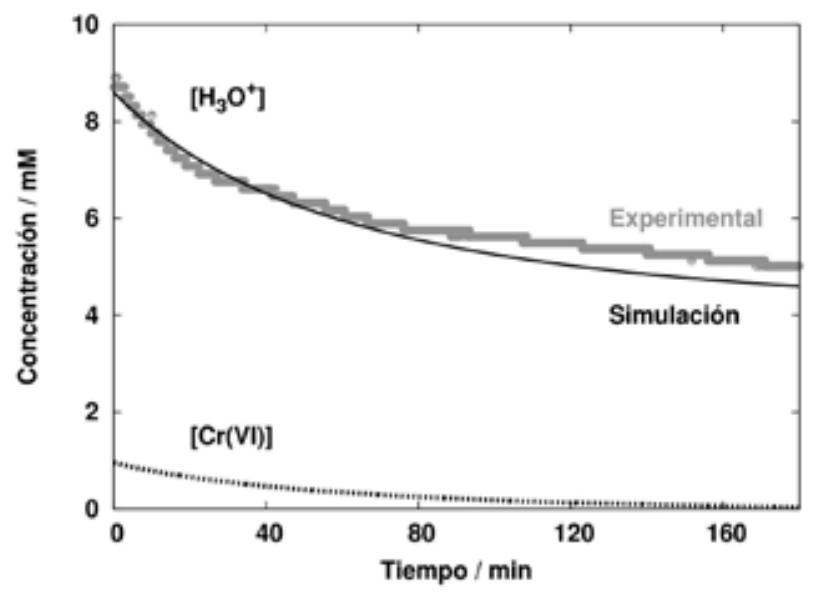

Figura 4. Resultados de la simulación numérica del mecanismo de reacción de la tabla 6 . Se comparan los datos experimentales de la medición de la concentración de iones $\mathrm{H}_{3} \mathrm{O}^{+}$con los obtenidos mediante la simulación. Adicionalmente, se presenta la concentración de $\mathrm{Cr}(\mathrm{VI})$ en función del tiempo obtenida de la simulación.

La figura 4 muestra el perfil cinético, calculado con el modelo propuesto, para la concentración de $\mathrm{Cr}(\mathrm{VI})$ y demuestra que el modelo es útil y capaz de describir lo que sucede cuando se adsorbe $\mathrm{Cr}(\mathrm{VI})$ sobre compost de residuos de clavel. Esta información es valiosa (además del conocimiento básico del sistema) para futuras implementaciones del proceso de remoción de $\mathrm{Cr}(\mathrm{VI})$ en aguas contaminadas utilizando compost, por ejemplo, a nivel de planta piloto. 


\section{CONCLUSIONES}

Los resultados obtenidos en este trabajo sugieren que, en las condiciones utilizadas, el proceso de remoción de $\mathrm{Cr}(\mathrm{VI})$ afecta negativamente a los microorganismos presentes en el compost obtenido de residuos de cultivos de clavel de la sabana de Bogotá. Por tanto, el mecanismo mediante el cual se lleva a cabo el proceso de remoción es netamente fisicoquímico. Además, se pudo establecer que el mecanismo para la remoción del $\mathrm{Cr}(\mathrm{VI})$ consta de 4 pasos fundamentales: a) Protonación del compost, el cual sigue una cinética de orden 2; b) Adsorción del Cr(VI) sobre los sitios protonados del compost, que también es un proceso cinético de orden 2; c) Reducción del Cr(VI) a Cr(III) previa adsorción del Cr(VI) y d) Liberación del Cr(III) desde el compost hacia la disolución.

\section{AGRADECIMIENTO}

Los autores agradecen a la Dirección Nacional de Investigación (DIB) de la Universidad Nacional de Colombia, por la financiación a través del proyecto HERMES 12308.

\section{REFERENCIAS BIBLIOGRÁFICAS}

1. Hubbe MA, Hasan SH, Ducoste JJ. Cellulosic substrates for removal of pollutants from aqueous systems: A review. 1. Metals. BioRes. 2011; 6(2): 2161-2287.

2. Paradelo R, Barral MT. Evaluation of the potential capacity as biosorbents of two MSW composts with different $\mathrm{Cu}, \mathrm{Pb}$ and $\mathrm{Zn}$ concentrations. Bioresour Technol. 2012;104:810-813.

3. Vargas C, Brandão PFB, Ágreda J, Castillo E. Bioadsorption using compost: An alternative for removal of chromium (VI) from aqueous solutions. BioRes. 2012; 7(3): 2711-2727.

4. Vargas C, Carriazo JG, Castillo E. Estudio de materiales adsorbentes de bajo costo para remover $\mathrm{Cr}(\mathrm{VI})$ de efluentes acuosos. Ingeniería e Investigación. 2011; 31(1): 154-162.

5. Park D, Lim S-R, Yun Y-S, Park JM. Development of a new Cr(VI)-biosorbent from agricultural biowaste. Bioresour Technol. 2008; 99(18):8810-8818.

6. Miretzky P, Cirelli AF. Cr(VI) and Cr(III) removal from aqueous solution by raw and modified lignocellulosic materials: a review. J Hazard Mater. 2010;180(1-3):1-19.

7. Leofanti G, Padovan M, Tozzola G, Venturelli B. Surface area and pore texture of catalysts. Catal Today. 1998;41(1-3):207-19.

8. Barrena R, Vázquez F, Gordillo MA, Gea T, Sánchez A. Respirometric assays at fixed and process temperatures to monitor composting process. Bioresour Technol. 2005;96(10):1153-1159.

9. Li Y, Yue Q, Gao B, Li Q, Li C. Adsorption thermodynamic and kinetic studies of dissolved chromium onto humic acids. Colloids Surf B Biointerfaces. 2008;65(1):2529.

10. Radhakrishnan K, Hindmarsh A. Description and Use of LSODE, the Livermore solver 
for ordinary Differential equations. NASA Reference publication 1327. Lawrence Livermore National Laboratory. Report UCRL-ID-113855, 1993.

11. Boni MR, Sbaffoni S. The potential of compost-based biobarriers for Cr(VI) removal from contaminated groundwater: column test. J Hazard Mater. 2009;166(2-3):10871095.

12. Cimino G, Passerini A,Toscano G. Removal of toxic cations and Cr(VI) from aqueous solution by hazelnut shell. Water Res. 2000; 34: 2955-2962.

13. Agarwal GS, Bhuptawat HK, Chaudhari S. Biosorption of aqueous chromium(VI) by Tamarindus indica seeds. Bioresour Technol. 2006;97(7):949-956.

14. Wei YL, Lee YC, Hsieh HF. XANES study of Cr sorbed by a kitchen waste compost from water. Chemosphere. 2005;61(7):1051-1060.

15. Albadarin AB, Al-Muhtaseb AH, Al-laqtah NA, Walker GM, Allen SJ, Ahmad MNM. Biosorption of toxic chromium from aqueous phase by lignin: mechanism, effect of other metal ions and salts. Chem Eng J. 2011;169(1-3):20-30.

16. Mendoza J. Estudio termocinético de la oxidación de fenol con peróxido de hidrógeno catalizada en fase homogénea y heterogénea. [Tesis de maestría]. Bogotá: Universidad Nacional de Colombia; 2011. 